\title{
Correction to: Observations on the breeding biology of the Andean Ibis (Theristicus branickii) in Ecuador
}

\author{
Nivia Luzuriaga-Neira ${ }^{1} \cdot$ Diego Cuichán $^{2} \cdot$ Mathilde da Costa $^{1} \cdot$ Nathaly Reyes $^{1}$ \\ Published online: 8 April 2021 \\ (C) Sociedade Brasileira de Ornitologia 2021
}

\section{Correction to: Ornithol. Res.}

https://doi.org/10.1007/s43388-021-00044-3

This article was updated to correct Nivia Luzuriaga-Neira's name.

The online version of the original article can be found at https://doi.org/ 10.1007/s43388-021-00044-3

\section{Nivia Luzuriaga-Neira} nluzuriaga@uce.edu.ec

1 Unidad de Estudios de la Fauna Silvestre, Facultad de Medicina, Veterinaria y Zootecnia, Universidad Central del Ecuador, Jerónimo Leiton s/n y Gatto Sobral, 170521 Quito, EC, Ecuador

2 Reserva Ecológica Antisana, Ministerio del Ambiente de Ecuador, Calle Humboldt s/n y Av. Antisana,, Píntag, Ecuador 